\title{
Small Outbreak with a Big Impact: Post Cataract Bacillus Endophthalmitis Outbreak
}

\author{
Huma Majeed $^{1 *}$, Juneed M. Lanker ${ }^{2}$, Rubina Lone ${ }^{1}$ and Syed Arshi ${ }^{1}$ \\ ${ }^{1}$ Department of Microbiology, SKIMS MC, J\&K, India \\ ${ }^{2}$ Department of surgery, SKIMS, J\&K, India \\ *Corresponding author
}

\section{A B S T R A C T}

\begin{abstract}
Endophthalmitis is an event which is considered to be quite rare nowadays. There are many studies on post cataract endophthalmitis but very few studies have come up on post cataract Bacillus endophthalmitis and our study focuses on a similar event. Our aim was to evaluate an outbreak of post operative endophthalmitis which took place in a hospital in north Kashmir and lasted over a period of three weeks. Elective cataract surgery was performed on patients, all above 50 years of age with intra ocular lens implantation. Out of all these patients operated for cataract, 8 patients developed endophthalmitis. Vitreal tap was done and subjected to gram staining, $\mathrm{KOH}$ and wet mount preparations and culture. Samples from different areas and instruments of the ophthalmology unit were also taken. Out of the eight vitreous taps of the cases, three were positive. These three samples showed gram positive bacilli in singles and chains supported by cultures which also revealed Bacillus spp. Subsequently samples from different areas and instruments of the ophthalmology unit also revealed Bacillus spp. The coexistence of Bacillus spp with human flora, its image as a contaminant and its rare occurrence as a cause of endophthalmitis are the things which hinder its establishment as an etiological agent. But the thing which always has to be kept in mind is that Bacillus spp whenever cultured from ocular tissues or fluids should not be dismissed as contaminant and should always be investigated. This outbreak of Bacillus endophthalmitis was linked to contaminated ophthalmic instruments and other materials used during the surgery and in the post operative period. The key is recognition of sterilization and decontamination as a problem, raising awareness, providing practical training and resources for the same.
\end{abstract}

\section{Introduction}

Endophthalmitis is an event which is considered to be quite rare nowadays. Mostly endophthalmitis is exogenous in origin $(95 \%)$ i.e. due to trauma or intraocular surgery. (Gribomont, 2009) It is one of the most devastating complications of intraocular surgery.
Endophthalmitis is the inflammatory response to a bacterial, fungal, or parasitic invasion of the eye. (Gribomont, 2009) Bacterial endophthalmitis is a blinding eye disease that can result from either exogenous (postoperative, post-traumatic) or endogenous (hematogenous) causes. The outcome of the infection varies with the microbial agent involved and the rapidity of response to 
treatment. Studies have shown that endophthalmitis, where the causative agents include normal flora from oral cavities, nasal cavities and skin is of a low grade type and treatment is usually successful. In contrast, endophthalmitis due to environmental organisms like Bacillus spp is more resistant to handle and is quickly devastating for ocular structures (Das et al., 2001).

Post-operative endophthalmitis is a rare but feared complication after cataract surgery and enucleation can be rare sequelae (Roger F. Steinert) Endophthalmitis caused by Bacillus sppis rare, and most cases are related to trauma (Das et al., 2001; Foster et al., 1996; Barletta and Small, 1996; Roy et al., 1997). There are many studies on post cataract endophthalmitis butvery few studies have come up on post cataract Bacillus endophthalmitis (Simini, 1998) and our study focuses on a similar event. What could have been the reasons and the preventive measures for it.

The present study aimed to evaluate an outbreak of post-operative endophthalmitis which took place in a hospital in north Kashmir and lasted over a period of three weeks.

\section{Materials and Methods}

Elective cataract surgery was performed on patients, all above 50 years of age with intra ocular lens implantation. Patients were operated in the ophthalmology theatre. In the post-operative period within 2-5 days patients complained of redness, pain, declining visual acuity in the operated eyes.

Out of all these patients operated for cataract, 8 patients developed endophthalmitis. Median time for presentation of post-operative endophthalmitis has been reported to be 3-10 days with majority presenting within two weeks (Roger F. Steinert). Vitreal taps were performed and intra vitreal and systemic antibiotics were given. Vitreal taps are considered to be better samples (Jack J. Kanski and Brad Bowling, 2011) and the probability of finding a microorganism by direct examination or by culture is indeed higher in the vitreous (40 to $69 \%$ of the cases) than in the aqueous humour (Gribomont, 2009). The samples were collected from those patients only, who presented with features of endophthalmitis.

This vitreal tap was subjected to gram staining, $\mathrm{KOH}$ and wet mount (Edward J. Bottone, 2010) preparations. Part of the sample was directly plated on blood agar and maconkey agar and part of the sample was inoculated in BHI broth for sub-culturing.

Repeated samples from different areas and instruments of the ophthalmology unit were also taken over a period of three weeks and culture of the same was performed.

\section{Results and Discussion}

An outbreak of endophthalmitis developed over a period of three weeks. Eight patients out of all the patients who were operated for cataract, had features of endophthalmitis. The patients developed acute endophthalmitis over a period of 2-5 days postoperatively. Patients had symptoms of redness, decreased vision and pain in the operated eye. It was associated with systemic symptoms of fever, malaise and leukocytosis.

Out of the eight vitreous taps of the cases, three were positive. These three samples showed gram positive bacilli in singles and chains on direct gram staining. Wet preparation of the vitreous fluid showed motile bacilli. Culture of the same vitreous taps grew Bacillus spp. Gram-stained smears prepared from it revealed uniform bacillary 
morphology with oval, centrally situated spores.

On blood agar at $37^{\circ} \mathrm{C}$, colonies were dull grey and opaque with a rough matted surface. Colony perimeters were irregular and swarming in character, likely due to swarming motility. Zones of beta-hemolysis were present. Rests of the five cases of endophthalmitis were culture negative.

Subsequently samples from different areas and instruments of the ophthalmology unit were taken over a period of three weeks and culture of the same was performed. This was what it revealed.

Our study is based on a small outbreak in an ophthalmology unit in a hospital in north Kashmir. The occurrence of two or more similar cases relating to place and time is identified as a cluster or an outbreak. (Guidelines on Prevention and Control of Hospital Acquired Infections)

Studies show very low rates of incidence of post cataract surgery endophthalmitis like 0.040\% (Wrong et al.,), $0.030 \%$ (Miller et al., 1997), 0.17\% (Menikoff et al.,; Roger F. Steinert)

In one of the studies, Bacillus spp was mentioned as a causative agent in $22 \%$ cases of traumatic endophthalmitis whereas in $0 \%$ cases of postoperative endophthalmitis (Ashok Nataraj, 2010). Our study had cases of postoperative endophthalmitis but the causative agent was Bacillus spp which is a rare cause for the same. Bacillus species of all kinds as a cause of postoperative endophthalmitis has been reported only rarely. (Simini, 1998; Wai-Man Chan et al., 2003)

Out of 8 cases of endophthalmitis only 3 vitreous samples showed gram positive bacilli on grams staining (37.5\%). Gram positive bacilli detected on vitreous microscopy have to be taken as Bacillus spp unless proved otherwise (Das et al., 2001). The same three vitreous samples grew Bacillus spp on culture (37.5\%). Approximately $64 \%$ of eyes with clinical endophthalmitis will display a positive culture (Ashok Nataraj, 2010). Although in our study lesser percentage of endophthalmitis cases were positive on culture but it has been documented that negative cultures don't rule out infection (Jack J. Kanski and Brad Bowling, 2011).

A virulent micro-organism should be suspected when the endophthalmitis occurs around 48 hours after cataract surgery (Gribomont, 2009) and that is what we were looking for. Although from our research we established the causative organism to be Bacillus spp but from its cultural characteristics, high virulence of the Bacillus spp which caused the infection in our patients, its refractoriness to antibiotic administration and poor visual outcome (loss of eyesight) we assumed our organism to be Bacillus cereus (Don B. David et al., 1994).

B. cereus endophthalmitis is a devastating malignant eye infection because of the rapidity with which the infection progresses and the bacterium's elaboration of a multitude of virulence factors (Edward J. Bottone, 2010). Drobniewski detailed the results of 35 cases of B. cereus endophthalmitis reported during a century, of which 20 eyes were lost to enucleation and one was lost to blindness (Edward J. Bottone, 2010; Drobniewski, 1993).

Despite antibiotic administration and surgical intervention Bacillus cereus results in the loss of eye very quickly with possible need of enucleation. This is supplemented by the fact that even if the antibiotics render the eye sterile, ocular damage continues to occur due to toxin production (Das et al., 2001). This 
intervention window is extremely narrow for B. cereus; in addition, there may be irreversible and progressive intraocular damage. (Wai-Man Chan et al., 2003)

Very few culture-confirmed cases of postoperative B. cereus endophthalmitis in the English-language literature (Wai-Man Chan et al., 2003; Roy et al., 1997; Orsi et al., 1999; Kunimoto et al., 1999) have been reported.

The prognosis remains dismal in spite of recent advances. The most important reason for this includes a delay in diagnosis and virulent spectrum of organisms. (Ashok Nataraj, 2010)

The prognosis is poor once a diagnosis of Bacillus spp endophthalmitis is established (Hemady et al., 1990). Bacillus spp was regarded as a "contaminant" when isolated from a clinical specimen but any Bacillus spp whenever cultured from ocular tissues or body fluids should not be dismissed as contaminants. (Hemady et al., 1990; Edward J. Bottone, 2010)

Table.1

\begin{tabular}{|l|l|l|l|}
\hline SAMPLE & CULTURE & SAMPLE & CULTURE \\
\hline IOL jars & Sterile & Scissors & Bacillus spp \\
\hline Dressing pads of theatre & Sterile & Irrigation aspiration bulb & Bacillus $\mathrm{spp}$ \\
\hline Betadine & Sterile & Trolley & Bacillus $\mathrm{spp}$ \\
\hline Theatre eye sheet & Sterile & Eye pads & Bacillus $\mathrm{spp}$ \\
\hline $\begin{array}{l}\text { Surgical instrument } \\
\text { trays }\end{array}$ & Sterile & Microscope attachments & Bacillus $\mathrm{spp}$ \\
\hline Gloves & Sterile & Linen sheets & \\
\hline Microscope & Sterile & Gown & Bacillus spp \\
\hline Forceps & Bacillus spp & Face mask & Bacillus $\mathrm{spp}$ \\
\hline Dressing pad of ward & Bacillus $\mathrm{spp}$ & Surgical instruments & Bacillus spp \\
\hline OT table surface & Bacillus $\mathrm{spp}$ & & \\
\hline
\end{tabular}

This outbreak of Bacillus endophthalmitis was linked to contaminated ophthalmic instruments and other materials used during the surgery and in the post-operative period. It was because Bacillus spp was yielded on cultures from the same (Table).

Besides flora of the eye, contaminated solutions and instruments have been considered to be potential sources of endophthalmitis. Contamination of sterilized instruments, disposable supplies, surgical field, or the intraocular lens all have been reported (Jack J. Kanski and Brad Bowling, 2011; Donzis et al., 1988). Epidemic clusters of endophthalmitis have resulted from these types of external contaminations (William
Lloyd Clark, 2016) as has resulted in our case. B. cereus nosocomial infections by contaminated fomites such as gauze, linens, and ventilators, etc., may serve as the source of the B. cereus outbreak (Edward J. Bottone, 2010; Simini, 1998). B. cereus endophthalmitis due to contaminated needles, injection paraphernalia, illicit drugs (Grossniklaus et al., 1985; Shamsuddin et al., 1982) or iatrogenic methods has been mentioned in many studies (Edward J. Bottone, 2010; Kerkenezov, 1953). It is conceivable that other items, e.g., alcohol sponges and gloves etc could also have been contaminated (Kerkenezov, 1953).

Roy et al., (1997) reported the largest case 
series of Bacillus endophthalmitis related to cataract extraction; 14 eyes were infected as a result of intraocular use of bacteriacontaminated viscoelastic material during the procedure (Wai-Man Chan et al., 2003; Roy et al., 1997).

Now the reasons which could be attributed to this post-operative Bacillus endophthalmitis outbreak could be improper cleaning of instruments, resistant organism and CSSD failure.

Keeping instruments free from gross soiling and minimizing the time between instruments leaving the operating rooms and cleaning will reduce the risk of biological material drying in the grooves and crevices (Reducing Public Health Risks Associated with Reusable Medical Devices). Among instruments considered as difficult to clean includes some ophthalmic instruments also like aspirators, phaco needles and tubings (Reducing Public Health Risks Associated with Reusable Medical Devices).

Improper washing of instruments prior to sterilizing could be a more important cause than faulty sterilization. Care is required with both washing the instruments and autoclaving them, as the latter is never absolute.

So both matters need to be investigated if there is an ongoing epidemic of postoperative endophthalmitis (ESCRS Guidelines on Prevention).

Spores of Bacillus are particularly troublesome because spores can be refractory to pasteurization and gamma radiation, and their hydrophobic nature allows them to adhere to surfaces. (Edward J. Bottone, 2010)

It has been pointed out that Bacillus spores can survive multiple heat disinfection treatments as well as chemical disinfection systems used for the minimum recommended lens care techniques (Edward J. Bottone, 2010; Kunimoto et al., 1999). CSSDs have a significant contribution to the quality of services provided by healthcare facilities and have an impact on public health, patient safety, infection control and thus needs to be strengthened.

Despite their supposed rare occurrence as cause of endophthalmitis, Bacillus spp is an environmentally pervasive bacterium coexisting with human flora. The major obstacle in evaluating its presence when isolated from a clinical specimen is overcoming its dubious image as a contaminant.

But the thing which always has to be kept in mind is that Bacillus spp whenever cultured from ocular tissues or fluids should not be dismissed as contaminant (Hemady et al., 1990; Edward J. Bottone, 2010) and should always be investigated.

Reprocessing of medical devices is common worldwide due to cost constraints and nonavailability of adequate number of devices. There is under reporting of infections/ outbreaks due to inadequate decontamination of medical devices (Nizam Damani, 2014). This leads to infections during surgery and spread of MDR organisms. Issues which need to be put forward are; decontamination in resource limited countries; what are the possibilities. The way forward is that it should be a continuous process of improvement based on many small steps rather expecting a revolutionary innovation.

There should be a structured approach towards quality improvement which includes recognition of sterilization and decontamination as a problem, raising awareness, providing practical training and resources, implementing good practices and 
audit, and keeping a documentation of the same.

\section{References}

Ashok Nataraj. Post Traumatic Endophthalmitis, Kerala Journal of Ophthalmology, Sept. 2010. XXII (3), Pg 258-261.

Barletta JP, and Small KW. Successful Visual Recovery in Delayed Onset Bacillus Cereus Endophthalmitis. Ophthalmic Surg Lasers. 1996; 27: 70-2.

Das T, Choudhary K, Sharma S, Jalali S, Nuthethi R. Clinical Profile and Outcome in Bacillus Endophthalmitis, Kanuri Santhama Retina Vitreous Centre, LV Prasad Eye Institute, Hyderabad,Tpd@Ivpeye.Stph.Net

Das T, Choudhury K, Sharma S, Jalali S, Nuthethi R. Clinical Profile and Outcome in Bacillus Endophthalmitis. Endophthalmitis Research Group. Ophthalmology. 2001 108: 1819-25.

Don B. David, Graham R Kirkby, Bruce A Noble. Bacillus Cereus Endophthalmitis, British journal of ophthalmology 1994; 78: 577-580.

Donzis, P. B., B. J. Mondino, and B. A. Weisman. 1988. Bacillus Keratitis with Contaminated Contact Lens Case System. Am. J. Ophthalmol. 105: 195197.

Drobniewski, F. A. 1993. Bacillus Cereus and Related Species. Clin. Microbiol. Rev. 6: 324-338

Edward J. Bottone, Bacillus Cereus, a Volatile Human Pathogen, Clinical Microbiology Reviews, Apr. 2010, 23(2): 382-398.

ESCRS Guidelines on Prevention, Investigation and Management of PostOperative Endophthalmitis. www.Escrs.Org/Vienna2011/Programm e/Handouts/IC-100/IC.
Foster RE, Martinez JA, Murray TG, Rubsmen PE, Flynn HW, Forster RK. Useful Visual Outcomes after Treatment of Bacillus Cereus Endophthalmitis. Ophthalmology. 1996. 103: 390-7.

Gribomont, A.C. Post-Cataract Surgery Endophthalmitis: An Update. Bull. Soc. Belge Ophthalmol, 2009. 311, 43-49.

Grossniklaus, H., H. Bruner, W. E. Frank, and E. W. Purnell. 1985. Bacillus cereus Panophthalmitis Appearing as an Acute Glaucoma in a Drug Addict. Am. J. Ophthalmol. 100: 334.

Guidelines on Prevention and Control of Hospital Acquired Infections, Pg-40p, WHO

Hemady, R., M Zaltas, B Paton, C F Foster, A $S$ Baker, British Journal of Ophthalmology 1990; 74(1): 26-9

Jack J. Kanski and Brad Bowling. Lens. Clinical Ophthalmology; 2011 Elsevier, 7: 289-291

Kerkenezov, N. Panophthalmitis after a Blood Transfusion. Br. J. Ophthalmol. 1953. 37: 632-636.

Kunimoto DY, Das $\mathrm{T}$, Sharma $\mathrm{S}$, et al., Microbiologic Spectrum and Susceptibility of Isolates. I. Postoperative Endophthalmitis. Am J Ophthalmol 1999; 128:240-2.

Nizam Damani, 2014. WHO Revised CSSD Manual and Guidelines, Processing Medical Devices.

Orsi GB, Aureli P, Cassone A, Venditti M, Fara GM. Post-Surgical Bacillus Cereus Endophthalmitis Outbreak. J Hosp Infect 1999; 42: 250-2.

Reducing Public Health Risks Associated with Reusable Medical Devices, NCCTG, Australia, Pg; 18, 21, 22. Http:/Www.Tga.Gov.Au

Roger F. Steinert, Cataract Surgery, 3ed, Elsivier, David A, Eichensaum, Robert I Park, Post-Operative Endophthalmitis. 671-672 
Roy M, Chen JC, Miller M, Boyaner D, Kasner O, Edelstein E. Epidemic Bacillus Endophthalmitis After Cataract Surgery. Ophthalmology 1997; 104: 1768-72.

Shamsuddin, D., C. V. Tuazon, C. Levy, and J. Curtin. 1982. Bacillus Cereus Panophthalmitis: Source of the Organism. Rev. Infect. Dis. 4: 97-103.

Simini, B. Outbreak of Bacillus Cereus Endophthalmitis in Rome. Lancet. 1998. 351: 1258
Wai-Man Chan, 1, 2 David T. L. Liu, 1 Carmen K. M. Chan, 2 Kelvin K. L. Chong, 1 and Dennis S. C. Lam 1, 2. Infective Endophthalmitis Caused by Bacillus Cereus after Cataract Extraction Surgery, Clinical Infectious Diseases 2003; 37: E31-34

William Lloyd Clark, MD; Chief Editor: Hampton Roy Sr, Postoperative Endophthalmitis, 2016.

\section{How to cite this article:}

Huma Majeed, Juneed M. Lanker, Rubina Lone and Syed Arshi. 2018. Small Outbreak with a Big Impact: Post Cataract Bacillus Endophthamitis Outbreak. Int.J.Curr.Microbiol.App.Sci. 7(08): 3147-3153. doi: https://doi.org/10.20546/ijcmas.2018.708.336 